\title{
Radioactivité dans le lait maternel et contamination de l'environnement*
}

\author{
G. CAMPOS VENUTI"**, S. RISICA $A^{\star \star}, S$. SIMULA

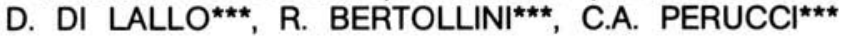

(Manuscrit reçu le 18 janvier 1988)

\begin{abstract}
RÉSUMÉ
Après l'accident de Tchernobyl, nous avons entrepris un programme de contrôle de la radioactivité artificielle dans le lait maternel, dans la ville de Rome. Cette recherche s'est déroulée en 1986 et en 1987 et est encore en cours. Nous avons confronté les concentrations de césium 134 et 137 mesurées avec celles du lait de vache et du lait en poudre. La dose efficace a été calculée pour les nourrissons.
\end{abstract}

En 1987, nous nous étions assigné un double objectif: 1) vérifier la représentativité des mères qui ont fourni les échantillons par rapport à toutes les mères du Latium; 2) prélever des échantillons dans le Latium et aussi dans d'autres régions de l'Italie du Nord. Nous visons donc à vérifier l'homogénéité de la contamination du lait maternel dans la région de Rome ainsi qu'à évaluer cette contamination dans les régions les plus frappées par les retombées de Tchernobyl.

\section{ABSTRACT}

Following the Chernobyl accident, monitoring of artificial radioactivity in breast milk in the city of Rome was organised. The research, developed during 1986 and 1987, is still continuing. Cesium 134 and 137 concentrations were compared with those of cow's and powdered milk. The effective dose for breast-fed infants was calculated. In 1987, the research was improved in two directions: 1) verifying whether the mothers involved in the milk collection were representative of the mothers' population of the whole Latium district; 2) collecting samples from the Latium district and some areas of Northern Italy, too. The aims were to verify the homogeneity of the breast milk contamination in the whole District, and to assess this contamination in the areas more heavily struck by the Chernobyl fallout.

* Communication présentée lors du congrès franco-italien SFRP-Associazione italiana di protezione contro le radiazioni, Castelgandolfo (Italie), 12-13 octobre 1987.

** Laboratorio di Fisica, Istituto Superiore di Sanità, Viale Regina Elena, 299, I 00161, Roma.

*** Osservatorio Epidemiologico della Regione Lazio, Roma. 


\section{INTRODUCTION}

Nous avons entrepris un programme de recherche sur la radioactivité dans le lait maternel, dans la ville de Rome, à la suite de la diffusion sur l'Italie du nuage de Tchernobyl; ces recherches se sont déroulées en 1986 et 1987. L'objectif était double:

a) mesurer, dans un but exclusivement sanitaire, la concentration de radionucléides artificiels émetteurs $\gamma$ dans ce produit si précieux afin d'évaluer ainsi l'exposition due à l'ingestion et la dose engagée pour les nourrissons;

b) par l'étude de ce même produit, évaluer l'évolution de la contamination humaine en fonction du temps.

Au fil des mois, nous avons développé ce programme en augmentant les lieux de prélèvement des échantillons, et en regroupant les données portant sur les caractéristiques socio-biologiques des femmes qui ont fourni des échantillons de leur lait.

Nous avons effectué la spectrométrie gamma de tous ces échantillons (450 ml environ), au moyen de deux cristaux de germanium H.P. coaxiaux; l'un a une efficacité de $38,2 \%$, un pouvoir de résolution en énergie de $1,95 \mathrm{keV}$; l'autre présente une efficacité de $26,6 \%$, un pouvoir de résolution en énergie de $1,73 \mathrm{keV}$.

\section{CONTAMINATION DU LAIT MATERNEL DANS LA VILLE DE ROME}

Les échantillons nous ont été fournis par:

- la banque du lait du nouvel hôpital "Regina Margherita" (à laquelle appartiennent cet hôpital, mais aussi une dizaine de cliniques et d'hôpitaux de la ville de Rome);

- la polyclinique Umberto $1^{\mathrm{er}}$;

- plus occasionnellement, la polyclinique Gemelli.

L'échantillon est un mélange composé de lait prélevé sur cinq à dix femmes, durant la première semaine suivant leur accouchement.

La figure 1 donne les concentrations de césium 137 et de césium 134 dans ces échantillons, entre mai 1986 et la fin de septembre 1987. On trouvera [1] la concentration en iode 131 dans la période initiale. On remarque une augmentation rapide de la concentration des deux césiums durant les premiers mois; l'augmentation est ensuite plus lente, jusqu'à la fin du premier semestre de 1987. Pendant le second semestre de 1987, la concentration des radionucléides diminue nettement. Le rapport initial ${ }^{137} \mathrm{Cs} /{ }^{134} \mathrm{Cs}$ est égal à 2 (compte tenu des écarts types), puis il augmente lentement, et de manière continue, à cause de la période du ${ }^{134} \mathrm{Cs}$.

Les concentrations mesurées sont faibles, qu'il s'agisse des valeurs absolues, ou des valeurs comparées aux contaminations simultanées par d'autres produits employés dans la première enfance. En particulier, la concentration dans le lait maternel est bien inférieure à celle existant dans le lait de vache fourni par la centrale de Rome. 


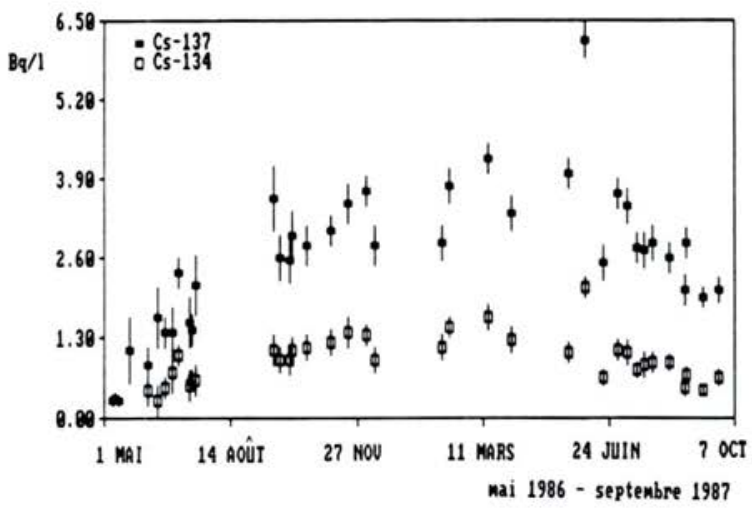

Fig. 1. - Concentrations de ${ }^{137}$ Cs et ${ }^{134}$ Cs dans le lait maternel (hôpitaux de Rome). Les barres représentent deux écarts type.

La figure $2[4,6]$ donne les concentrations de ${ }^{137} \mathrm{Cs}$ et ${ }^{134} \mathrm{Cs}$ dans le lait de la centrale de Rome, de mai 1986 à septembre 1987: la valeur maximale de ${ }^{137} \mathrm{Cs}$ est plus de vingt fois supérieure à celle trouvée dans le lait maternel. Dans le lait de vache, il y a une nouvelle augmentation de la contamination à la fin de l'été 1986: elle est due à la nourriture du bétail par du fourrage coupé en mai. A partir d'avril 1987, on constate le début d'une nette diminution.

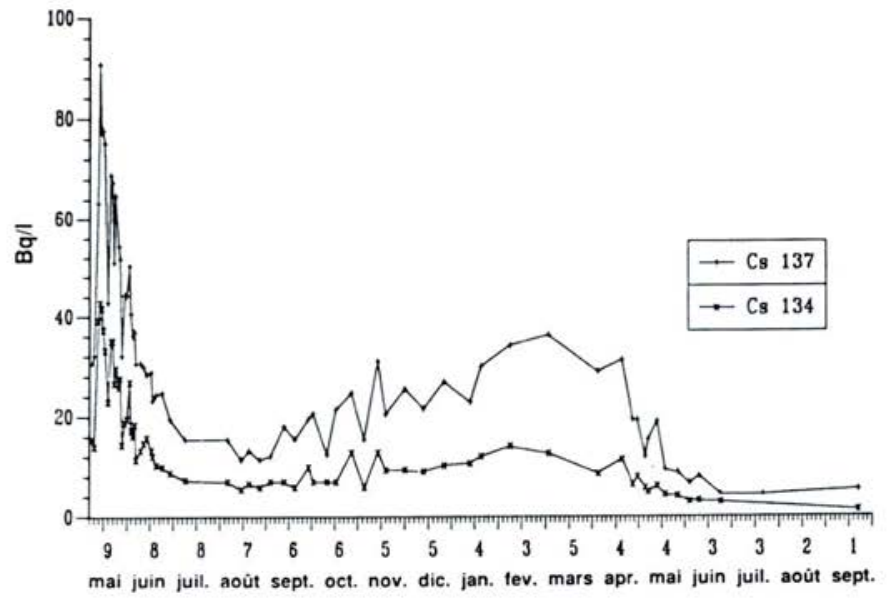

Fig. 2. - Concentrations de ${ }^{137} \mathrm{Cs}$ et ${ }^{134} \mathrm{Cs}$ dans le lait (Centrale de Rome, mai 1986 - septembre 1987), d'après $[4,6]$. 
A côté du lait maternel et du lait de vache, une troisième nourriture pour la première enfance est le lait en poudre. Nous avons donc effectué une comparaison au moyen de toutes les données de contamination radioactive du lait en poudre que nous avons pu trouver. Voici les résultats de 740 analyses (de mai 1986 à juin 1987) de lait en poudre vendu en Italie:

- concentration de ${ }^{137} \mathrm{Cs}$ bien inférieure à $37 \mathrm{~Bq} \mathrm{~kg}{ }^{-1}$ dans $78 \%$ des échantillons;

- concentration de ${ }^{137} \mathrm{Cs}$ comprise entre 40 et $190 \mathrm{~Bq} \mathrm{~kg}^{-1}$ dans $19 \%$ des échantillons;

- concentration de ${ }^{137} \mathrm{Cs}$ comprise entre 200 et $410 \mathrm{~Bq} \mathrm{~kg}^{-1}$ dans $3 \%$ des échantillons ( 1 seul échantillon a une concentration supérieure à $370 \mathrm{~Bq} \mathrm{~kg}{ }^{-1}$ ).

De ces données, nous tirons deux conclusions:

a) dans la majeure partie des cas, le lait en poudre n'a été que très faiblement contaminé: c'est particulièrement important pour la dose collective des enfants;

b) dans le lait maternel, la concentration est comparable à celle des laits en poudre les plus faiblement contaminés.

Nous avons calculé l'équivalent de dose efficace engagé pour les enfants allaités au sein dans trois cas: naissance à la fin d'avril 1986; naissance à la fin d'octobre 1986; naissance à la fin de mars 1987. Pour être prudent, le modèle que nous avons adopté est un allaitement étalé sur six mois et une ingestion de 0,9 litre de lait par jour [5].

Utilisant les facteurs de conversion du NRPB [8], le calcul conduit, dans ces trois cas, aux doses efficaces suivantes: 14,11 et $8 \mu$ Sv. Dans ce calcul, nous avons même tenu compte de la contribution de l'iode 131 durant la période initiale: elle est de $8 \mu \mathrm{Sv}$ [1]. En utilisant des facteurs de conversions plus conservatoires (par exemple, ceux que la CIPR est en train d'élaborer) les doses obtenues sont 3-4 fois plus élevées et le rôle de l'iode n'est pas aussi important. C'est pendant cette première période qu'ont été atteintes les valeurs maximales à cause de la contribution de l'iode 131, bien que les mesures de restriction imposées en Italie sur le lait frais et les végétaux aient certainement considérablement diminué la dose due à l'iode 131 pour les femmes qui étaient enceintes en mai. L'équivalent de dose efficace est très faible pour les trois groupes de nouveaux-nés sélectionnés.

\section{VÉRIFICATION DE LA REPRÉSENTATIVITÉ DES ÉCHANTILLONS}

Pour contrôler que les niveaux de radioactivité mesurés dans le lait des femmes qui ont participé à cette étude sont représentatifs de ceux du lait de toutes les femmes qui ont allaité, dans le Latium, durant la même période, il aurait fallu confronter certaines caractéristiques socio-biologiques de ces deux groupes. Comme il n'existe pas, en Italie, de données fiables sur les femmes en allaitement, nous avons employé deux méthodes de comparaison indirecte:

a) Nous avons vérifié la similitude des caractéristiques des femmes ayant accouché dans les hôpitaux où nous avons prélevé des échantillons, et celles des autres nourrices. Grâce aux données de l'Observatoire 
d'épidémiologie [2] sur le système de contrôle des naissances dans le Latium, nous avons effectué une comparaison des deux groupes de mères, portant sur le poids de l'enfant à la naissance, sur l'âge de la mère, sur le nombre d'accouchements, sur le temps de gestation, sur la gemellité, etc. Les figures 3 et 4 permettent de comparer l'âge des mères et le poids de l'enfant à la naissance. II n'y a pas de différence significative concernant ces variables, comme d'ailleurs concernant les autres.

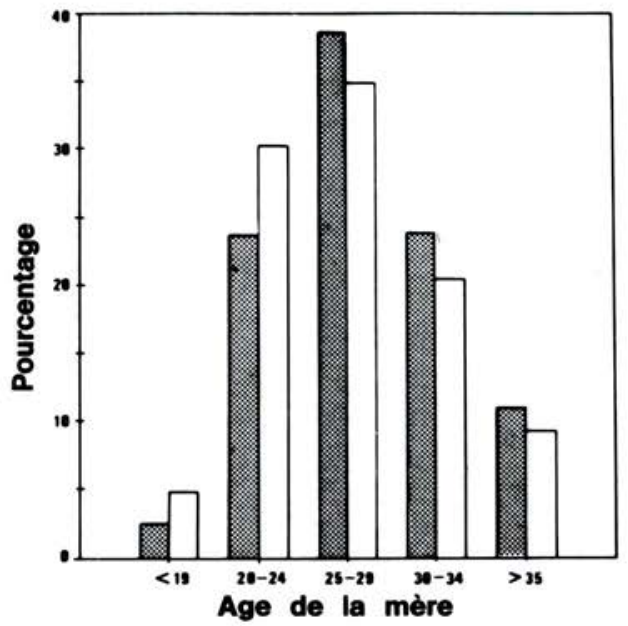

Fig. 3. - Répartition de l'âge des parturientes dans les centres de collecte

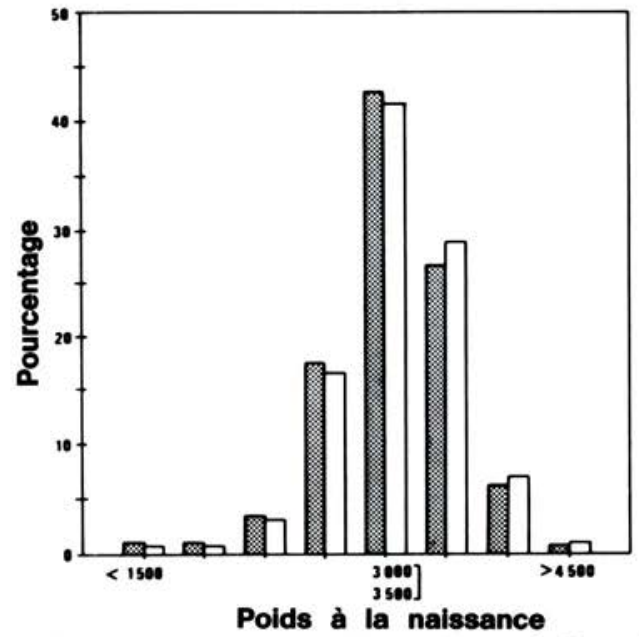

Fig. 4. - Répartition du poids des enfants à la naissance dans les centres de collecte (intervalle: $500 \mathrm{~g}$ ). 
b) Nous avons employé les informations individuelles fournies par un formulaire, rempli par les femmes nous ayant donné du lait, pour effectuer une comparaison avec l'ensemble de la population maternelle de la région. Nous disposons des données concernant 110 femmes ayant allaité entre mars et septembre 1987. Les figures 5 et 6 permettent de comparer cet échantillon à l'ensemble des mères habitant le Latium en ce qui concerne l'âge et le niveau d'instruction. II n'y a pas de différence significative statistiquement en ce qui concerne la répartition selon l'âge; cependant, les femmes de plus de 35 ans sont moins nombreuses à allaiter. Cette différence ne peut s'expliquer que par une tradition culturelle: l'âge ne produit pas un effet négatif prouvé sur la capacité féminine de donner du lait en quantité suffisante [3].

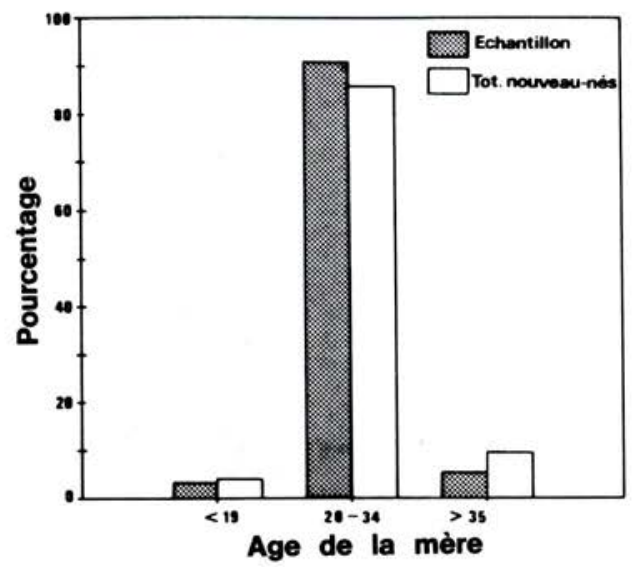

Fig. 5. - Nombre de nouveaux-nés en fonction de l'âge de la mère. Comparaison de l'échantillon étudié à l'ensemble du Latium.

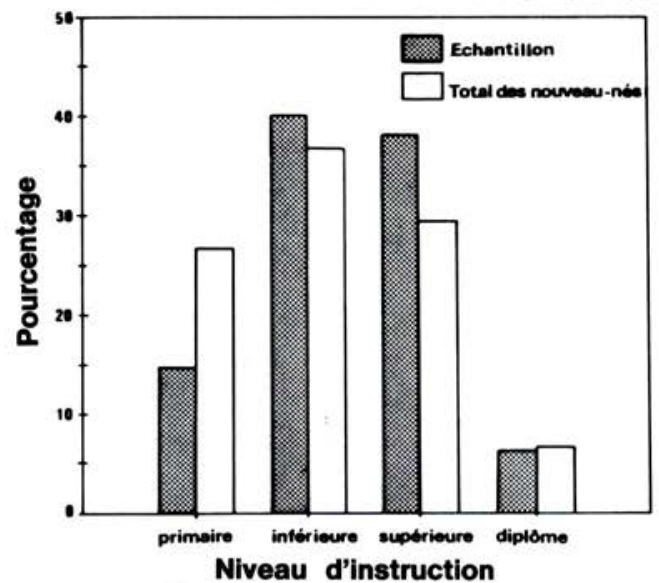

Fig. 6. - Nombre de nouveaux-nés en fonction du degré d'instruction de la mère.

Comparaison de l'échantillon étudié à l'ensemble du Latium. 
Dans notre échantillon, les femmes possédant un niveąu d'instruction élémentaire sont, en valeur relative, moins nombreuses. C'est statistiquement remarquable: les données globales dont nous disposons datent de 1981 [7], alors que notre échantillon est de 1987. Dans l'intervalle, le niveau d'instruction a, peut être, changé, ce qui expliquerait la différence. II ne faut pas oublier, cependant, que d'autres enquêtes [9] ont montré qu'il existe une corrélation entre le niveau d'instruction et l'allaitement au sein.

\section{ELARGISSEMENT TERRITORIAL DE LA RECHERCHE}

Ainsi que nous le laissions entendre dans l'introduction, nous avons programmé, au début de 1987, un élargissement territorial de cette recherche. Nous souhaitons d'abord confirmer (ou non), par la mesure d'échantillons venant d'autres zones du Latium, l'hypothèse [1] que les valeurs des concentrations trouvées dans les échantillons de lait de Rome représentaient bien les niveaux de l'ensemble de la région. Notre second objectif est de vérifier le rapport existant entre la radioactivité artificielle dans le lait maternel, et la contamination de l'environnement, en mesurant des échantillons venant des régions de l'Italie du nord les plus contaminées par le nuage.

A cet effet, nous avons intégré dans notre recherche deux maternités des provinces de Viterbo et Latina, et la maternité de Trento. Le lait est un produit précieux; il est difficile d'en obtenir de grandes quantités, et nous n'avons pu mesurer qu'un nombre limité d'échantillons. La comparaison avec les autres hôpitaux du Latium montre le bien fondé de l'hypothèse que les mesures faites sur les échantillons de Rome fournissent une représentation assez exacte de la situation dans la région; par ailleurs, les données venant de Trento indiquent qu'apparemment les niveaux dans le lait maternel ne sont qu'un peu plus élevés dans une région beaucoup plus frappée par la contamination radioactive. Ce dernier résultat est, en apparence, étrange; il peut $\mathrm{y}$ avoir deux explications possibles:

a) cette comparaison a été effectuée un certain temps après l'accident: les contributions principales à la dose due à l'ingestion résultaient alors d'aliments venant de l'ensemble du territoire national (viande, pain, pâtes) et pas uniquement de provenance locale (lait, légumes, fruits), ainsi que cela était le cas dans la phase initiale;

b) dans les régions les plus frappées, les femmes enceintes ont fait beaucoup plus attention à ne pas consommer de denrées pouvant être contaminées.

Les résultats quantitatifs précis concernant cet élargissement territorial seront publiés ultérieurement.

\section{CONCLUSION}

Les échantillons que nous avons analysés jusqu'à présent nous conduisent aux conclusions suivantes:

1. Les niveaux de radioactivité mesurés provenant d'un échantillon de mères, sont représentatifs de l'ensemble des femmes du Latium qui ont allaité après l'accident de Tchernobyl; 
2. Pour les nourrissons du Latium, les doses dues à l'iode 131, au césium 134 et au césium 137 dans le lait maternel, dans les mois suivant l'accident, sont faibles, et inférieures à celles correspondant à une nourriture par du lait de vache ou du lait en poudre;

3. Plus de dix mois après l'accident, la contamination du lait maternel n'est apparemment pas influencée significativement par la distribution géographique des retombées; nous vérifions actuellement ce résultat en mesurant des échantillons provenant d'autres régions italiennes.

II sera nécessaire de faire des analyses supplémentaires pour vérifier l'hypothèse qu'il est possible d'utiliser le taux de contamination du lait maternel comme indice d'exposition de la population dans son ensemble.

\section{REMERCIEMENTS}

Les auteurs remercient Mlle Paola DI CIACCIO pour son travail de traduction.

\section{BIBLIOGRAPHIE}

[1] BERTOLLINI R., CAMPOS VENUTI G., DI LALLO D., PERUCCI C.A., RISICA S., SIMULA S. Radioactivity in breast milk in central Italy in the aftermath of Chernobyl. Acta Paediatr. Scand., 1987, 76, 530-531.

[2] BERTOLLINI R., DI LALLO D., DI LENA P., GITARELLI D., RAPITI E., PERUCCI C. Sistema di sorveglianza della natalità e mortalità infantile nel Lazio, Anno 1984. Progetto Salute, 7, 1987.

[3] DEWEY K.G., FINLEY D.A., STRADE M.A. and LONNERDAL B. Relationship of maternal age to breast milk volume and composition. In: Human lactation 2 (Hamosh M. and Goldman A.S., Eds.) New York: Plenum Press, 1986.

[4] GIROLAMI B. Communication personnelle.

[5] INTERNATIONAL COMMISSION ON RADIOLOGICAL PROTECTION (ICRP). Report of the Task Group on reference man (ICRP Publication N. 23). Oxford: Pergamon press, 1974.

[6] ISTITUTO SUPERIORE DI SANITÀ (ISS). II rischio ambientale nella produzione di energia: risultati sperimentali, calcoli e riflessioni dopo Chernobyl. (Risica S., Ed.) Ann. ISS, 1987, 23 (2) 177-522.

[7] ISTAT. Statistiche sanitarie, 1986, vol. 27.

[8] NATIONAL RADIOLOGICAL PROTECTION BOARD (NRPB). Committed doses to selected organs and committed effective doses from intakes of radionuclides. Report NRPB-GS7, 1987, confirmé par Revised generalised derived limits for radioisotopes of strontium, iodine, caesium, plutonium, americium and curium. NRPB-GS8, 1987.

[9] Report of the task force on the assessment of the scientific evidence relating to infant-feeding practices and infant health. Pediatrics 1984, 74, suppl. 4. 